\title{
Evaluation of Assembly Part Build Orientation in Additive Manufacturing Environment using Data Envelopment Analysis
}

\author{
Kasin Ransikarbum ${ }^{1 a}$, Rapeepan Pitakaso ${ }^{1}$, and Namhun Kim ${ }^{2}$ \\ ${ }^{1}$ Department of Industrial Engineering, Ubonratchathani University, Thailand \\ ${ }^{2}$ Department of System Design and Control, Ulsan National Institute of Science and Technology, South Korea
}

\begin{abstract}
Whereas Subtractive Manufacturing (SM) is a process by which 3D objects are constructed by cutting material away from a solid block of material, such as milling and lathe machine; Additive Manufacturing (AM) is a synonym for 3D printing and other processes by which 3D objects are constructed by successively depositing material in layers. Recently, AM has become widespread for both industrial and personal use thanks to the freedom and benefits it provides in designing parts, reducing lead time, improving inventory, and supply chain. However, few studies examine process planning issues in AM. In addition, existing studies focus on production of an individual part alone. In this study, we examine the assembly orientation alternatives' efficiency using Data Envelopment Analysis (DEA) technique for different AM technologies and their associated materials under conflicting criteria. A case study of hardware fasteners using bolt and nut fabrication is illustrated in the study. Our results show that different AM technologies and materials clearly impact efficiency of part production and thus suggest optimal orientation in AM process planning platform.
\end{abstract}

\section{Introduction}

Recently, emphasis in the Additive Manufacturing (AM) community has moved from rapid prototyping towards rapid manufacturing for end-use parts. It refers to a set of technologies used to produce end-use parts directly from 3D Computer-Aided Design (CAD) models by additively building them in layers. Several studies suggest advantages and challenges related to AM [1-6]. For example, according to Wohlers [1], while advantages of the AM include reduction of tooling, agile manufacturing, decentralized supply chain, and reduction in inventory and part consolidation; challenges are noted for cost of machines and materials, quality assurance, and traditional attitudes of customers. The American Society for Testing and Materials (ASTM [7]) has standardized AM technologies into seven main categories: 1) Photopolymer vat, 2) Material extrusion, 3) Powder bed fusion, 4) Directed energy deposition, 5) Sheet lamination, 6) Material jetting, and 7) Binder jetting.

Researchers have discussed future of AM business and supply chain [8-10]. Rylands et al. [8] suggested that AM in the future will not replace, but compliment and strength traditional manufacturing. Esmaeilian et al. [9] pointed out that future process planning of manufacturing systems is not limited to production planning, but includes all aspects of a manufacturing firm, such as capacity planning and scheduling. Ransikarbum et al. [10] discuss the AM supply chain's strategic, tactical, and operational levels for planning, in which a production stage includes technology selection, part orientation and location, and part-to-printer scheduling problems.
Concerning issues in process planning, Gallagher [11] pointed out that there is a need to assist designers and practitioners in selecting the most feasible AM technology within the manufacturing process of parts. Conflicting criteria include build speed, build material, surface finish, part size, production cost, reliability, waste disposal, employee training and skill requirement, local availability of technology, etc. [12-13]. In addition, the optimal part orientation is considered to be a critical issue of AM processes as it can impact many key characteristics in part production, including processing cost and time, mechanical properties, and support volume [14-16]. In this study, we examine part orientation problem by investigating assembly part production from two key AM technologies; Fused Deposition Modeling (FDM) and Stereolithography (SLA). Evaluated criteria in the study are material consumption, process time, production cost, waste and support, part accuracy, assembling, and surface quality. Next, data are collected and analyzed using Data Envelopment Analysis (DEA) to evaluate part efficiency.

\section{Methodology}

Multi-Criteria Decision Analysis (MCDA) is a subdiscipline of operations research and management science (OR/MS) that explicitly considers multiple criteria in a decision-making environment [17]. It is mainly used to support decision-makers facing decision and planning problems that a unique optimal solution does not exist and decision-maker's preferences are involved. Common MCDA methods including Analytic Hierarchy Process (AHP), Data Envelopment Analysis (DEA), Machine

\footnotetext{
${ }^{\text {a }}$ Corresponding author: kasinphd@gmail.com
} 
Learning, Multi-Objective Programming (MOP), and Goal Programming (GP) have been developed and used in a variety of applications (e.g., [18-21]). DEA has been well-known in many applications as an efficiency measurement technique that measures the relative efficiency of decision making units (DMUs) with multiple inputs and outputs, without the need for predefined production functions or assumptions. Generally, the model produces an efficiency score (between zero and 1) for each unit, in which a unit with a score of $100 \%$ is relatively efficient and any unit with a score of less than $100 \%$ is considered as relatively inefficient.

\section{a) Mathematical Model for DEA}

The DEA model formulated as a linear programming (LP) problem can be shown in Eqs (1)-(4).

$$
\text { Maximize Efficiency } \quad \sum_{j \in J} y_{j, k_{0}} V_{j}
$$

$$
\begin{aligned}
& \text { Subject to: } \quad \sum_{i \in I} x_{i, k_{0}} U_{i}=1 \\
& \qquad \begin{array}{l}
\sum_{j \in J} y_{j, k} V_{j}-\sum_{i \in I} x_{i, k} U_{i} \leq 0 \quad ; \forall k \in K \\
U_{i} \geq 0 ; \forall i \in I ; V_{j} \geq 0 ; \forall j \in J
\end{array}
\end{aligned}
$$

\section{Experimental Design and Case Study}

In contrast to an individual part production commonly used in other existing studies (e.g., [4]-[5]), we illustrate the case study of hardware fasteners (i.e., bolt and nut) simultaneously built on a printer platform in this study. Initially, assembly are designed using SolidWorks software as shown in Fig. 1. Next, a designed experiment is conducted based on different AM technologies, material types associated with each AM printer, process criteria, and orientation of assembly (Table 1).

Table 1. Factors and levels for an experiment

\begin{tabular}{ll}
\hline \hline \multicolumn{1}{c}{ Factor } & \multicolumn{1}{c}{ Levels } \\
\hline AM technology & 1) Fused Deposition Modeling (FDM); 2) Stereolithography (SLA) \\
Material Type & 1) Acrylonitrile butadiene styrene (ABS); 2) Polylactic acid (PLA); 3) Resin \\
Process Criteria & 1) Material; 2) Time; 3) Cost; 4) Waste; 5) Accuracy; 6) Assembling; 7) Surface \\
Orientation & 1) $0^{\circ}$ angles; 2) $45^{\circ}$ angles; 3) 90 $90^{\circ}$ angles; 4) $180^{\circ}$ angles \\
\hline \hline
\end{tabular}

\subsection{AM Technology}

Two AM processes considered in this study are FDM and SLA. FDM is a type of material extrusion in an AM process, in which material is selectively dispensed through a nozzle as the extrusion head or the build platform moves in the $x-y$ plane. On the other hand, SLA uses an ultraviolet laser and $\mathrm{x}-\mathrm{y}$ scanning mirrors to scan the top surface of a vat of photopolymer resin called photopolymerization.
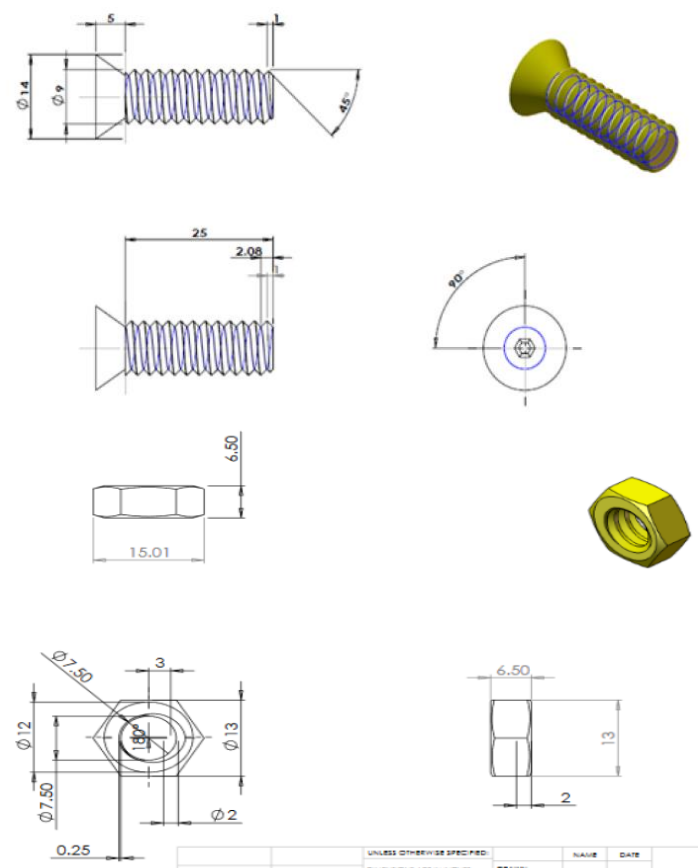

Figure 1. Case Study of an assembly part

\subsection{Process Criteria}

\subsubsection{Material consumption (Gram)}

Consumption of the material for SLA (i.e., liquid resin) is computed directly from what is used in gram unit. On the other hand, material for FDM for both ABS and PLA is in the form of filament. Thus, filament diameter and material density are used to convert material length in meter unit to compute volume in gram unit for a comparative study.

\subsubsection{Process time (Minute)}

Build time is related to the time spent on layer scanning, which is dependent on the number of slices. Whereas process time for SLA can be measured directly from the built time of the printer, FDM requires time to heat the printer platform and time to cool down in addition to the build time.

\subsubsection{Production cost (Baht)}

Production cost refers to the resources consumed during the manufacturing of a part in AM, which usually contains direct and indirect cost. In this study, material cost is used for direct cost, while energy and labor are used for indirect cost.

\subsubsection{Waste and Support (Gram)}

Support structure is needed in some particular AM processes, such as FDM and SLA for over-hangings. 


\subsubsection{Part accuracy (Millimeter)}

Parts accuracy refers to the difference between the produced part and the designed model. Part orientation can affect both shrinkage and distortion, which are the main factors in AM resulting in this difference. In this study, part accuracy is measure in an average error between the diameters of part design and actual printed part.

\subsubsection{Assembling (Scale 1-10)}

The assembly part (i.e., bolt and nut) are evaluated for the completeness of assembling using qualitative measurement from expert opinions, which are converted to quantitative scale (10- the best; 1-the worst).

\subsubsection{Surface quality (Scale 1-10)}

Parts which are typically parallel or perpendicular to the build orientation tend to have a better surface roughness than those whose face normal has an angle to the build direction. We perform qualitative measurement for this criterion (10- the best; 1 -the worst).

\section{$4 \quad$ Results and Discussion}

\subsection{Assembly part fabrication}

We fabricate assembly parts at the UBonratchathani University 3D printing Lab (UBU3D Lab) (Fig.2). In particular, FDM printer specifications are da Vinci 1.0 from XYZ Printing firm with $18.4 * 20 * 22$ inch in platform capacity and single extruder with diameter 0.4 $\mathrm{mm}$. Next, SLA printer specifications are $5 * 5 * 7.9$ inch in platform capacity with $0.3 \mathrm{~mm}$ resolution. The summary of the data from the designed experiment are illustrated in Table 2.

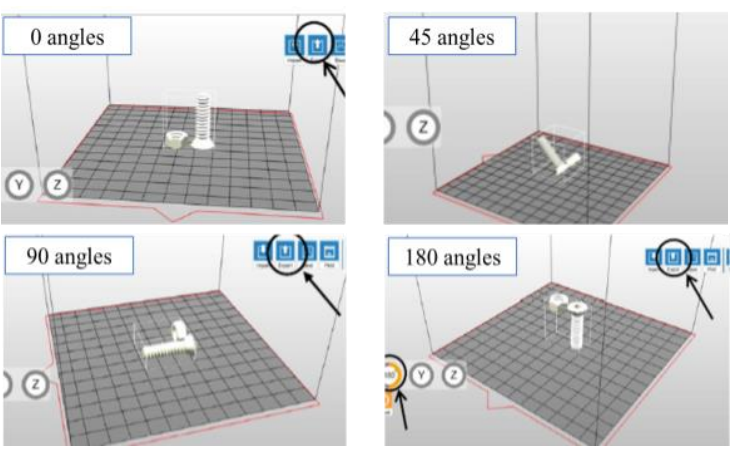

Figure 2. Orientation/Direction setting for all printers

Table 2. Results for all designed criteria

\begin{tabular}{lllll}
\hline \multicolumn{1}{c}{ Criteria } & \multicolumn{3}{c}{ Data } \\
\hline Orientation & $0^{\circ}$ angles & $45^{\circ}$ angles & $90^{\circ}$ angles & $180^{\circ}$ angles \\
FDM: ABS & & & & \\
Material (g.) & 1.69 & 2.56 & 2.65 & 2.47 \\
Time (Min.) & 32 & 51 & 58 & 36 \\
Cost (Baht) & 82 & 133 & 151 & 95 \\
Support (g.) & 0.00 & 0.87 & 0.95 & 0.78 \\
Accuracy (mm) & 0.53 & 0.43 & 0.57 & 0.39 \\
Assembling & 9 & 8 & 8 & 6 \\
Surface quality & 5 & 6 & 5 & 6 \\
FDM: PLA & & & & \\
Material (g.) & 2.24 & 2.88 & 2.85 & 2.36 \\
Time (Min.) & 21 & 31 & 21 & 31 \\
Cost (Baht) & 58 & 85 & 58 & 83 \\
Support (g.) & 0.00 & 0.64 & 0.61 & 0.12 \\
Accuracy (mm) & 0.57 & 0.43 & 0.43 & 0.70 \\
Assembling & 7 & 2 & 3 & 3 \\
Surface quality & 6 & 6 & 6 & 6 \\
SLA: Resin & & & & \\
Material (g.) & 1.78 & 2.41 & 4.15 & 2.73 \\
Time (Min.) & 68 & 78 & 81 & 65 \\
Cost (Baht) & 185 & 215 & 238 & 186 \\
Support (g.) & 0.00 & 0.63 & 2.37 & 0.95 \\
Accuracy (mm) & 0.34 & 0.51 & 0.47 & 1.00 \\
Assembling & 9 & 8 & 8 & 9 \\
Surface quality & 9 & 2 & 9 & 9 \\
\hline \hline
\end{tabular}

\subsection{DEA-based relative efficiency}

We now illustrate the DEA analysis for multiple input and output based on evaluated criteria to obtain efficiency of each part orientation alternative. In this study, we model the linear programming model of DEA in LINDO software. In this study, input criteria are material consumption, process time, production cost, and waste/support; whereas output criteria are part accuracy, assembling, and surface condition, respectively. In addition, three key sets of DEA analyses are performed based on different material types for each AM technology. That is, the first set of DEA analysis for ABS material of FDM printer contains four Decision Making Units (DMUs) and we report the results for each orientation direction (i.e., DMUs 1-4); the second set of DEA 
analysis for PLA material of FDM printer contains another four DMUs (i.e., DMUs 5-8); and the third set of DEA analysis for resin material of SLA printer contains the last four DMUs (i.e., DMUs 9-12). The relative efficiency scores for all orientation alternatives are reported in Table 3 .

Table 3. Relative efficiency score for each alternative

\begin{tabular}{ccccc}
\hline \hline AM / Material & $0^{\circ}$ angles & $45^{\circ}$ angles & $90^{\circ}$ angles & $180^{\circ}$ angles \\
\hline FDM / ABS & 1.00 (DMU 1) & 1.00 (DMU 2) & 0.63 (DMU 3) & 1.00 (DMU 4) \\
FDM / PLA & 1.00 (DMU 5) & 1.00 (DMU 6) & 1.00 (DMU 7) & 0.92 (DMU 8) \\
SLA / Resin & 1.00 (DMU 9) & 0.77 (DMU 10) & 0.84 (DMU 11) & 1.00 (DMU 12) \\
\hline \hline
\end{tabular}

DEA analysis provides insights regarding efficiency of each alternative whether it is efficient when multiple inputs and outputs are considered, simultaneously. Results show that efficiency score for FDM/ABS of $0^{\circ}$ angles, $45^{\circ}$ angles, and $180^{\circ}$ angles are 1.00 being superior to $90^{\circ}$ angles; whereas efficiency score for FDM/PLA of $0^{\circ}$ angles, $45^{\circ}$ angles, and $90^{\circ}$ angles are 1.00 being superior to $180^{\circ}$ angles. That is, different materials on the same AM process show to impact efficiency of process planning. Next, when SLA printer is used, results show that $0^{\circ}$ angles and $180^{\circ}$ angles are superior to other angles. In addition, across all the orientation alternatives of assembly part of all printer types, fabrication with $0^{\circ}$ angles appears to be the only efficient alternative with 1.00 .

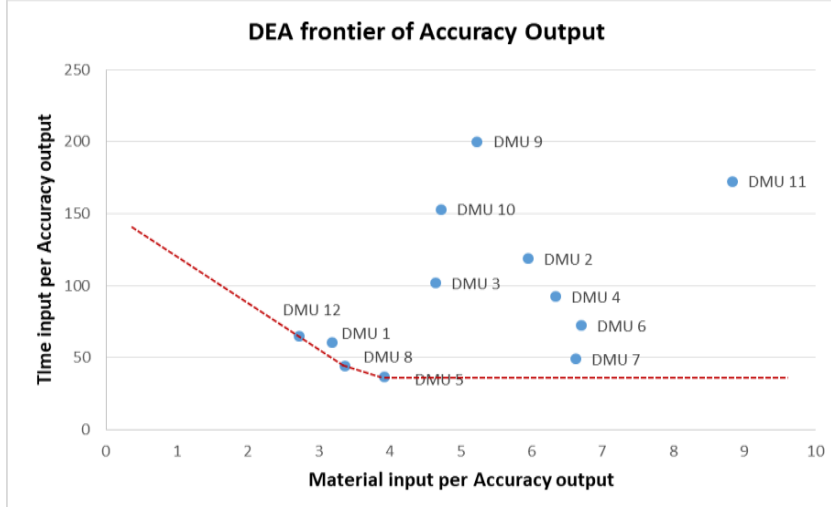

Figure 3. Illustration of the efficient front

\subsection{Insights on efficiency frontier}

Previously, when all the input and output criteria are considered simultaneously, efficiency score for all decision making units (DMUs) can be computed. Now, we illustrate the efficiency frontier when two arbitrary input criteria are considered against one output criterion. Efficient frontier is useful to visualize the plot of efficient units for any pair of input variables against an output variable. For example, supposed that a decision maker is more interested in the time and material input as well as interested in the accuracy output. In this case, the performance ratios for all DMUs can be computed, such that each input criterion is divided by the output criterion of interest. Thus, the x-axis in Fig. 3 shows standardized usage of material per accuracy, whereas the y-axis shows standardized usage of time per accuracy in our study.

By plotting all the standardized usage of all DMUs in the graph, it can be seen that DMU 5 (i.e., FDM with PLA at $0^{\circ}$ angles) and DMU 12 (i.e., SLA with Resin at $180^{\circ}$ angles) are efficient. In addition, any points connecting these two DMUs are also efficient (i.e., DMU 8 of FDM with PLA at $180^{\circ}$ angles) with respect to the mix of use on these two inputs. We note that the visualization of DEA frontier can be implemented for other pairs of input criteria and other output criteria of interest as well.

\section{Conclusion and future research}

Additive manufacturing has potentially transformed how products are designed, produced, and serviced, which enables on-demand production and unlocks digital design tools. In this study, by investigating different AM technologies with available materials, our results showed that orientation directions not only affected individual criterion, but also an overall efficiency in process and production planning. In particular, results show that orientation directions of assembly part fabricated from 1) FDM/ABS of $0^{\circ}, 45^{\circ}$, and $180^{\circ}$ angles; 2) FDM/PLA of $0^{\circ}, 45^{\circ}$, and $90^{\circ}$ angles, and 3 ) SLA/Resin of $0^{\circ}$ and $180^{\circ}$ angles are efficient. Thus, fabrication with $0^{\circ}$ angles appears to be the only efficient alternative across all the printer types and materials. Future directions of this study are to evaluate the results found from this study with other types of AM technologies and materials as well as with other part and assembly applications. In addition, integrating other MCDA techniques to secure optimal alternative when preferences of decision makers are incorporated in DEA could also be investigated.

\section{Acknowledgement}

This work was supported in part by the Office of the Higher Education Commissions (OHEC) and the Thailand Research Fund (TRF) under research grant for new scholar (MRG): MRG6180181. The opinions expressed herein are those of the authors and do not necessarily reflect the views of the funding agencies.

\section{References}

1. T. Wohlers, "Wohlers report 2016, " Wohlers Associates, Inc.J.

2. I. Campbell, D. Bourell, I. Gibson, "Additive manufacturing: rapid prototyping comes of age," Rapid Prototyping Journal Vol. 18, 2012, pp. 255258.

3. S. Ha, K. Ransikarbum, H. Han, D. Kwon, N. Kim, and H. Kim, "A Dimensional Compensation Algorithm for Vertical Bending Deformation of 3D printed Parts in Selective Laser Sintering," Rapid Prototyping Journal, 2018, 24(6), pp. 955-963. 
4. K. Ransikarbum and N. Kim, "Data envelopment analysis-based multi-criteria decision making for part orientation selection in fused deposition modeling," Proceedings in International Conference on Industrial Engineering and Applications (ICIEA), April 21-23 2017, Nagoya, Japan, pp. 81-85.

5. K. Ransikarbum, N. Kim, "Multi-criteria selection problem of part orientation in 3D fused deposition modeling based on analytic hierarchy process model: A case study", Industrial Engineering and Engineering Management (IEEM), Singapore, 2017, IEEE International Conference on, pp. 1455-1459.

6. G. Manogharan, RA, Wysk, OLA, Harrysson, "Additive manufacturing-integrated hybrid manufacturing and subtractive processes: economic model and analysis," International Journal of Computer Integrated Manufacturing 2016, Vol. 29, pp. 473-488.

7. ASTM. Standard Terminology for Additive Manufacturing Technologies. ASTM International, West Conshohocken, PA, 2012.

8. B. Rylands, T. Böhme, R. Gorkin III, J. Fan, T. Birtchnell, "The adoption process and impact of additive manufacturing on manufacturing systems," Journal of Manufacturing Technology Management 2016, Vol. 27, pp. 969-989.

9. B. Esmaeilian B, S. Behdad, B. Wang, "The evolution and future of manufacturing: A review," Journal of Manufacturing Systems 2016, Vol. 39, pp. 79-100.

10. K. Ransikarbum, S. Ha, J. Ma, and N. Kim, "MultiObjective Optimization Model for Production Planning of the Build Chamber Utilization in Fused Deposition Modeling", Journal of Manufacturing Systems, 2017, Vol. 43, pp. 35-46.

11. A. Gallagher, "A Decision Support System Methodology For The Selection Of Rapid Prototyping Technologies For Investment-cast Gas Turbine Parts", Doctoral dissertation, University of Central Florida Orlando, FL, 2010.

12. S.H., Masood and A. Soo, "A rule based expert system for rapid prototyping system selection", Robotics and Computer-Integrated Manufacturing, 2002, Vol. 18, pp. 267-274.
13. K. Lokesh and P.K. Jain, "Selection of rapid prototyping technology", Advances in Production Engineering and Management, 2010, Vol. 5, pp. 7584.

14. J.A. Nelson, G. Galloway, A.E. Rennie, T.N. Abram, and G.R. Bennett, "Effects of scan direction and orientation on mechanical properties of laser sintered polyamide-12," International Journal of Advanced Design and Manufacturing Technology, 2014, Vol. 7, pp. 19-25.

15. Y. Zhang, A. Bernard, R.K. Gupta, and R. Harik, "Feature based building orientation optimization for additive manufacturing," Rapid Prototyping Journal, 2016, Vol. 22, pp. 358-376.

16. M. Taufik and P.K. Jain, "Role of build orientation in layered manufacturing: a review," International Journal of Manufacturing Technology and Management, 2013, Vol. 27, pp. 47-73.

17. M. Velasquez and P.T. Hester, "An analysis of multicriteria decision making methods," International Journal of Operations Research, 2013, Vol. 10, pp. 56-66.

18. K. Ransikarbum and S. Mason, "Multiple-objective analysis of integrated relief supply and network restoration in humanitarian logistics operations", International Journal of Production Research, 2016, Vol. 54(1), pp. 49-68.

19. K. Ransikarbum and S. Mason, "Goal programmingbased post-disaster decision making for integrated relief distribution and early-stage network restoration", International Journal of Production Economics, 2016, Vol. 182, pp. 324-341.

20. K. Ransikarbum, N. Kim, S. Ha, R.A. Wysk, and L. Rothrock, "A Highway-Driving System Design Viewpoint Using an Agent-Based Modeling of an Affordance-Based Finite State Automata," IEEE Access, 2018, Vol. 6, pp. 2193-2205.

21. Y. Oh, K. Ransikarbum, M. Busogi, D. Kwon, and N. Kim, "Adaptive SVM-based real-time quality assessment for primer-sealer dispensing process of sunroof assembly line," Reliability Engineering \& System Safety, 2019, 184, 202-212. 